\title{
BECN1 and BIM interactions with MCL-1 determine fludarabine resistance in leukemic B cells
}

\author{
A Sharma ${ }^{1,2}, \mathrm{~K}$ Singh ${ }^{1}, \mathrm{~S}$ Mazumder ${ }^{1}, \mathrm{BT} \mathrm{Hill}^{3}, \mathrm{M} \mathrm{Kalaycio}^{3}$ and A Almasan ${ }^{*, 1}$
}

The purine analog fludarabine $(\mathrm{Fd})$ is an essential therapeutic for chronic lymphocytic leukemia (CLL). Innate or acquired resistance to $\mathrm{Fd}$ is a significant clinical problem and is largely mediated by increased expression of BCL-2 family members. The antiapoptotic BCL-2 family proteins inhibit both apoptosis and autophagy, therefore, downregulation of antiapoptotic BCL-2 family proteins and enhanced autophagy must coexist in cells dying in response to an apoptosis inducing therapeutic. However, in the drug-resistant cells that have an increased dependence on antiapoptotic proteins, whether autophagy is also inhibited remains unclear. Here, we examined the role of the BCL-2 family in regulating cell death and autophagy in leukemic cell lines and their derivative isogenic Fd-resistant (FdR) cells. MCL-1 degradation following Fd treatment freed the proapoptotic effectors BIM and BECN1, thus leading to cell death-associated autophagy in Fd-sensitive cells. However, in FdR cells, low BIM expression and BECN1 sequestration by MCL-1 prevented cell death. Consistently, in sensitive cells inhibition of apoptosis using siBIM and of both the early-phase autophagy nucleation steps by siBECN1, shATG7 or 3-methyladenine and the late-phase autophagy by shLAMP2, significantly reduced Fd-induced cell death. Paradoxically, FdR cells were addicted to basal autophagy, which was dependent on AMP-activated protein kinase (AMPK) but not BECN1. Moreover, in FdR cells, inhibition of autophagy by shLAMP2, but not siBECN1, enhanced cell death. The BH3-mimetic obatoclax released BIM and BECN1 from MCL-1 in Fd-sensitive and BECN1 from MCL-1 in FdR cells, and was effective at killing both Fd-sensitive and - resistant leukemic cells, including primary CLL cells. Therefore, a differential regulation of autophagy through BECN1 and AMPK signaling in Fd-sensitive and - resistant cells determines the different possible outcomes of autophagy inhibition. These findings suggest effective means to overcome Fd resistance by induction of BIM-dependent apoptosis and activation of BECN1-dependent autophagy.

Cell Death and Disease (2013) 4, e628; doi:10.1038/cddis.2013.155; published online 16 May 2013

Subject Category: Cancer

Fludarabine $(\mathrm{Fd})$ is a nucleoside analog that is incorporated into the DNA and inhibits DNA/RNA synthesis and DNA repair. Therefore, apoptosis ultimately occurs in both proliferating and quiescent cells. ${ }^{1} \mathrm{Fd}$ is an essential component of therapy for chronic lymphocytic leukemia (CLL). ${ }^{2}$ However, primary or acquired resistance limits the effectiveness of this therapy. ${ }^{2}$ Thus, a mechanistic understanding of such resistance at the molecular level is urgently needed for the rational development of appropriate alternative or combination therapies in the clinic.

The BCL-2 family proteins that regulate apoptotic cell death mediate chemoresistance in various cancers, ${ }^{3}$ including CLL. ${ }^{4-6}$ The antiapoptotic members of this family, such as BCL-2, BCL-XL and MCL-1 inhibit apoptosis by binding to and inhibiting proapoptotic BCL-2 family proteins. ${ }^{7}$ The proapoptotic members include 'effector' and ' $\mathrm{BH} 3-o n l y$ ' proteins, which, in turn, include 'direct activators' and 'sensitizers'. Upon activation, the 'effector' proteins BAX and BAK oligomerize and form pores on the outer mitochondrial membrane to release cytochrome $c$ and subsequently lead to caspase activation and apoptosis. ${ }^{8,9}$ Activation of 'effector' proteins requires interaction with the 'direct activators', BIM and BID. 'Sensitizers', such as PUMA and NOXA interact with and prevent antiapoptotic proteins from interacting with BIM and BID. ${ }^{10}$

The functionally diverse BCL-2 family proteins, ${ }^{11}$ in addition to inhibition of apoptosis, also regulate autophagy, ${ }^{12,13}$ a catabolic process maintaining cellular turnover in both normal

\footnotetext{
${ }^{1}$ Department of Cancer Biology, Lerner Research Institute, Cleveland Clinic, Cleveland, OH, USA; ${ }^{2}$ Cleveland State University, Cleveland, OH, USA and ${ }^{3}$ Department of Hematologic Oncology and Blood Disorders, Taussig Cancer Institute, Cleveland Clinic, Cleveland, OH, USA

*Corresponding author: A Almasan, Department of Cancer Biology, Lerner Research Institute, Cleveland Clinic, NB40, 9500 Euclid Avenue, Cleveland, OH 44195, USA. Tel: +216 445 9970; Fax: +216 445 6269; E-mail: almasaa @ccf.org Keywords: autophagy; MCL-1; BECN1; BIM; fludarabine resistance

Abbreviations: AMPK, AMP-activated protein kinase; ATG7, autophagy-related protein 7; BECN1, Beclin; BIM, BCL-2-interacting mediator of cell death; Bcl-2, B-cell lymphoma 2; BIM, B-cell lymphoma 2 interacting mediator of cell death; Bcl-w, Bcl-2- like protein 2; Bcl-XL, B-cell lymphoma-extra large; BFL-1, BCL-2-related protein A1; Bax, Bcl-2-associated X protein; Bak, Bcl-2 homologous antagonist/killer; BH3, Bcl-2 homology domain 3; Bid, BH3-interacting domain death agonist; Bad, Bcl-2associated death promoter; CLL, chronic lymphocytic leukemia; Comp C, compound C; CQ, chloroquine; DAPI, 4',6-diamidino-2-phenylindole; Fd, fludarabine; FdR, fludarabine-resistant; IR, ionizing radiation; LAMP2, lysosomal-associated membrane protein 2; LC3, microtubule-associated protein 1 light chain 3 alpha; 3-MA, 3-methyladenine; MCL-1, myeloid cell leukemia sequence; NOXA, phorbol-12-myristate-13-acetate-induced protein 1; Ob, obatoclax; p62/SQSTM1, sequestosome 1; class III PI3K, class III phosphatidylinositol-3 kinase; PI, propidium iodide; PUMA, p53 upregulated modulator of apoptosis; PBS, phosphate buffered saline; MTS, 3-(4,5dimethylthiazol-2-yl)-5-(3-carboxymethoxyphenyl)-2-(4-sulfophenyl)-2H tetrazolium inner salt; sh, short-hairpin; ULK-1, Unc51-like kinase; Vps34, vacuolar protein sorting 34; Vps15, vacuolar protein sorting 15; PFT $\alpha$, Cyclic Pifithrin- $\alpha$ hydrobromide

Received 17.12.12; revised 03.4.13; accepted 10.4.13; Edited by H-U Simon
} 
and cancer cells. A double membrane vesicle 'autophagosome' initially forms around the target substrate and later fuses with lysosomes to form 'autolysosomes', where degradation takes place. ${ }^{14,15}$ The nucleation of the autophagosomal membrane is regulated by beclin1 (BECN1, ATG6), a $\mathrm{BH} 3-d o m a i n$ containing protein, which forms the core class III phosphatidylinositol-3 kinase (PI3K)-complex, BECN1/ Vps34/Vps15, that recruits essential autophagic proteins to a preautophagosomal membrane. ${ }^{12,14}$ The BCL-2 family proteins, BCL-2, BCL-XL and MCL-1 block autophagy by direct interaction and inhibition of BECN1. ${ }^{13,16,17}$

As autophagy can cause both cell death and survival, ${ }^{18-20}$ we investigated the molecular alterations of autophagy and BCL-2 family proteins in response to acquired chemoresistance. By comparing Fd-sensitive and - resistant (FdR) cells that were generated by chronic exposure to $\mathrm{Fd}$, we delineate how the drug-resistant cells adapt to chemotherapy by their ability to evade apoptosis by activating autophagy. Targeting alternative cell survival or cell death pathways could provide attractive treatment strategies.

\section{Results}

Fd induces autophagy and enhances autophagic flux. To study the regulation of Fd-induced cell death or acquired resistance by autophagy, we first examined Fd-induced autophagy using LC3 (also known as ATG8) processing as a marker of autophagy. As there are no Fd-sensitive CLL cell lines available, we chose pre-B leukemic cell lines as a Fdsensitive model (IC50 $\sim 10 \mu \mathrm{M})$, as reported in a previous study investigating autophagy. ${ }^{21}$ During autophagy, the 18-kDa cytosolic LC3-I is conjugated to phosphatidylethanolamine to form the 16-kDa LC3-II, which associates with the autophagosomal membrane. ${ }^{14,22} \mathrm{Fd}$ treatment increased LC3-II levels, as determined by immunoblotting, in both Nalm-6 and Reh cells (Figure 1a). Moreover, immunostaining analysis for LC3 in Nalm-6 cells treated with Fd for $1 \mathrm{~h}$ showed an increase in the number of LC3 puncta, indicating an enhanced autophagosome formation, $P<0.05$ (Figures $1 \mathrm{~b}$ and $\mathrm{c}$ ).

Next, the autophagic flux was examined to distinguish between regulation of synthesis versus degradation of LC3-I/II. ${ }^{14}$ To determine autophagic flux, chloroquine (CQ) was used to inhibit degradation through autophagy by blocking lysosomal acidification. ${ }^{14} \mathrm{CQ}$ pretreatment enhanced LC3 processing in both Nalm-6 and Reh (Figure 1d) and LC3 puncta in 4-h-Fd treated Nalm-6 cells, $P<0.05$ (Figures 1e and f). Additionally, the levels of p62/ SQSTM1 (known to be degraded by autophagy) ${ }^{14,23}$ decreased after Fd treatment, which was prevented by $\mathrm{CQ}$ pretreatment (Figure 1d). Thus, Fd induces autophagy and enhances autophagic flux in leukemic B cells.

Inhibition of autophagy prevents Fd-induced cell death. To determine whether autophagy regulated cellular response to $\mathrm{Fd}$ treatment, genetic and pharmacological approaches were used to inhibit autophagy. Short-hairpin (sh) RNAmediated knockdown of LAMP2, an essential lysosomal protein required for the late stages of autophagy, that is, autophagosome to lysosome fusion ${ }^{14,24}$ prevented basal
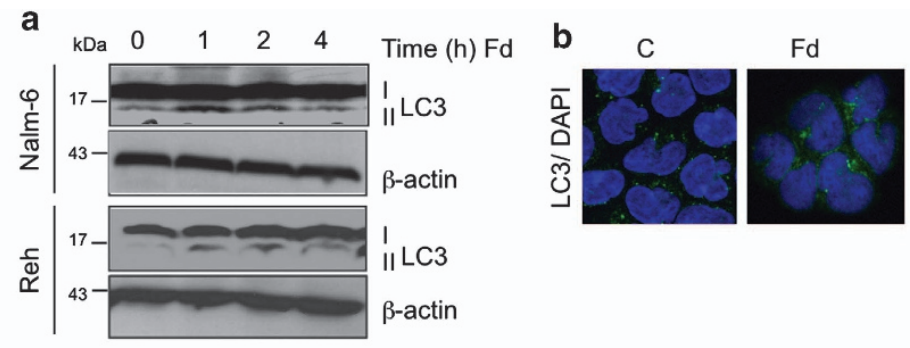

d

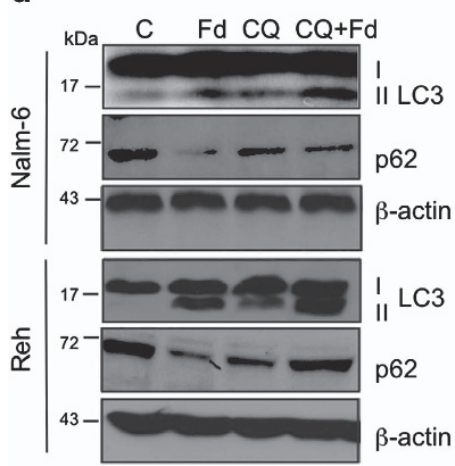

e

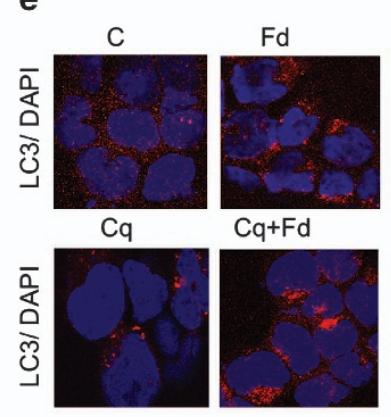

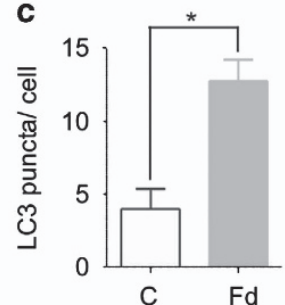

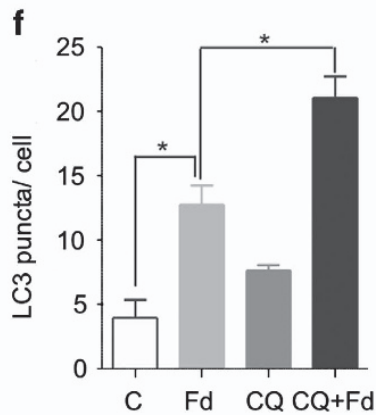

Figure $1 \mathrm{Fd}$ induces autophagy and enhances autophagic outflux.(a) Western blot for LC3 I/II expression, with $\beta$-actin serving as a loading control, in Nalm-6 and Reh cells treated with Fd for the indicated time. (b) Representative images of LC3 stained cytospins of Nalm- 6 cells treated with Fd for $1 \mathrm{~h}$. Nuclei were stained with DAPI. Image quantification of LC3 puncta is shown in (c). (d) Western blot for LC3 I/II, p62/SQSTM1(p62) and $\beta$-actin as a loading control in Nalm-6 and Reh cells pretreated with CQ for $1 \mathrm{~h}$ followed by 4-h treatment with Fd. (e) Confocal immunostaing for LC3-I/II in Nalm-6 cells pretreated with CQ for $1 \mathrm{~h}$ followed by $4-\mathrm{h}$ treatment with Fd. Nuclei were stained with DAPI. Quantification of LC3 puncta staining is shown in (f); ${ }^{*} P<0.05, n=3$ 
p62/SQSTM1 degradation in both Nalm-6 and Reh cells compared with their respective shControl-expressing cells (Figure 2c). Moreover, Fd-induced cell death was significantly reduced in both shLAMP2-expressing Nalm-6 $(P<0.001)$ and Reh $(P<0.01)$ compared with their respective shControl-expressing cells (Figure 2a). Similarly, in Nalm-6 cells expressing shRNA against ATG7 (Figure 2d), an essential autophagy protein that is required for LC3 processing, ${ }^{14,25}$ cell death decreased significantly $(P<0.05)$ (Figure 2b). Likewise, pharmacological inhibition of autophagy by 3-methyladenine (3-MA), known to inhibit the class III PI3K-complex required for nucleation of autophagosomes, ${ }^{14}$ also significantly prevented Fd-induced cell death in Nalm-6 cells $(P<0.05)$ (Figure $2 \mathrm{e})$. These findings suggest autophagy-associated cell death in response to $\mathrm{Fd}$ in leukemic cells.

Developing Fd resistance after chronic exposure. To determine how $\mathrm{Fd}$ resistance impacts on the therapeutic response of leukemic cells, we derived FdR cells. A 24-h-Fd treatment led to a significant dose-dependent decrease in MTS reduction in Nalm-6 $(P<0.0001)$ and Reh $(P<0.01)$ compared with no effect in the respective $\mathrm{FdR}$ cells (Figure 3a). Trypan blue staining showed $\sim 50 \%$ cell death in both Nalm-6 $(P<0.0001)$ and Reh $(P<0.001)$, compared with $<5 \%$ in the respective $\mathrm{FdR}$ cells following $24 \mathrm{~h}$ treatment (Figure 3b). Similarly, AnnexinV/PI staining showed $\sim 40-50 \%$ increase in cell death in Nalm-6, but not in the derivative $\mathrm{FdR}$ cells with $24 \mathrm{~h} \mathrm{Fd}$ treatment $(P<0.001)$ (Figure 3c). Interestingly, the FdR cells were specifically resistant to $\mathrm{Fd}$, but not bendamustine (a bifunctional purine analog and alkylating agent for CLL), ${ }^{2}$ that led to even higher induction of cleaved caspase-3 (Figure 3d) and higher dosedependent decrease in MTS reduction (Supplementary Figure S1) in FdR compared with Nalm-6 cells.

FdR cells are addicted to basal autophagy. Next, we compared the levels of autophagy between Fd-sensitive and - resistant cells. As in Figure 1, Fd treatment caused an induction of LC3-II in both Nalm-6 (Figure 4a) and Reh cells (Supplementary Figure S2a). However, in FdR cells, there was higher LC3-II in the untreated than in the respective parental cells (Figure 4a, Supplementary Figure S2a), suggesting a higher constitutive autophagy, which did not increase further with $\mathrm{Fd}$ treatment.

To further investigate whether higher LC3-II levels in FdR cells were due to elevated basal autophagy or inhibition of flux, ${ }^{14}$ we compared the autophagosome-lysosome fusion between Nalm- 6 and Nalm-6-FdR cells treated with Fd in the presence or absence of $\mathrm{CQ}$. As demonstrated by colocalization of LC3, a marker for autophagosomes, and LAMP2, a marker for lysosomes (Figures $4 \mathrm{~b}$ and c), CQ treatment alone caused an increased number of LC3 puncta, which colocalized with LAMP2 in Nalm-6 cells $(P<0.0001)$. Therefore, CQ inhibited autophagic degradation or flux through lysosomes. Additionally, Fd treatment alone of Nalm-6 showed a significant increase in LC3 puncta staining and LAMP2 colocalization compared with untreated Nalm-6 cells $(P<0.0001)$. Moreover, Fd treatment following $\mathrm{CQ}$ of Nalm-6 cells greatly enhanced both LC3 puncta staining and colocalization with LAMP2 $(P<0.0001)$, clearly indicating induction and completion of autophagy. In untreated FdR

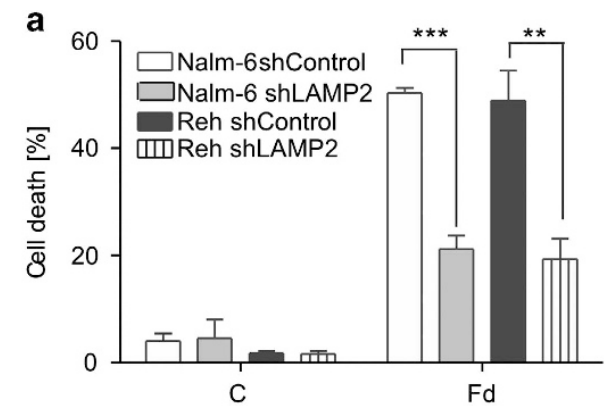

c

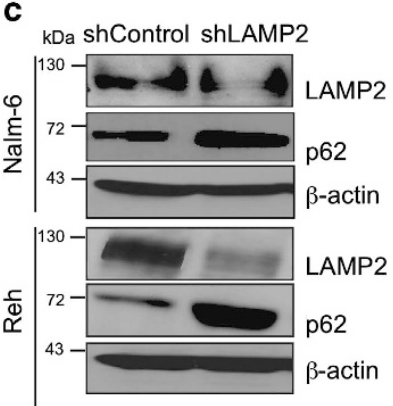

b

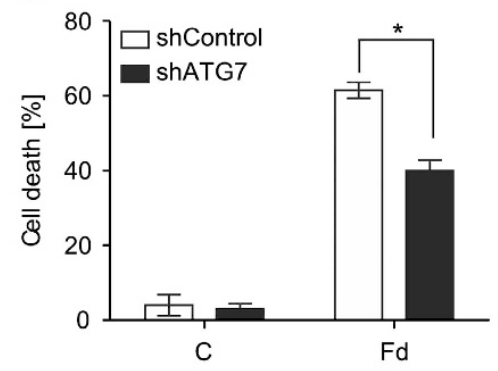

e kDashControlshATG7
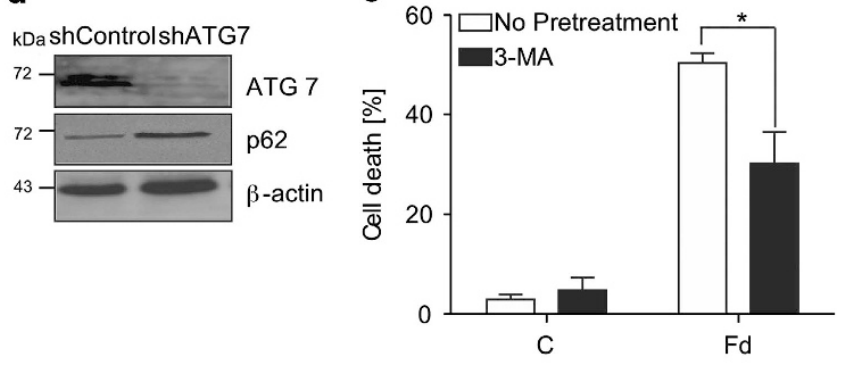

Figure 2 Inhibition of autophagy prevents Fd-induced cell death. (a) Cell death analysis in shControl and shLamp2-expressing cells in response to 24-h-Fd treatment as determined by trypan blue staining. (b) Cell death analysis in shControl and shATG7-expressing cells in response to 24 -h-Fd treatment as determined by AnnexinV/PI staining. (c) Immunoblotting for levels of LAMP2, p62 and $\beta$-actin in Nalm-6 and Reh cells expressing shControl or shLAMP2 lentiviral vectors. (d) Immunoblotting for levels of ATG7, p62 and $\beta$-actin in Nalm-6 cells expressing shControl or shATG7. (e) Nalm-6 cells were pretreated with 3-MA for $1 \mathrm{~h}$ followed by $24-\mathrm{h}$-Fd treatment and cell death was determined by AnnexinV/PI staining. All data are expressed as mean \pm S.D. of percentage of cell death; ${ }^{*} P<0.05,{ }^{\star \star} P<0.01,{ }^{* \star \star} P<0.001, n=3$ 


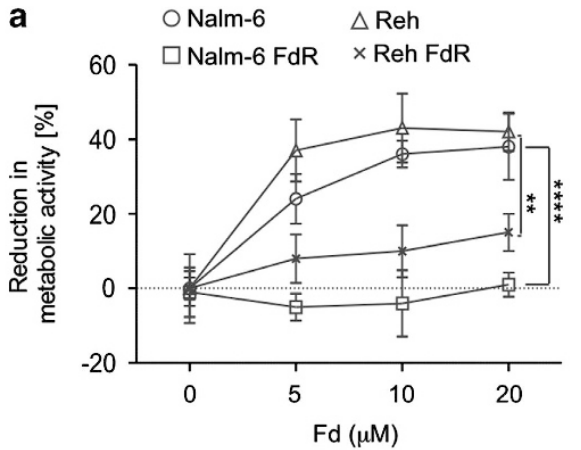

b
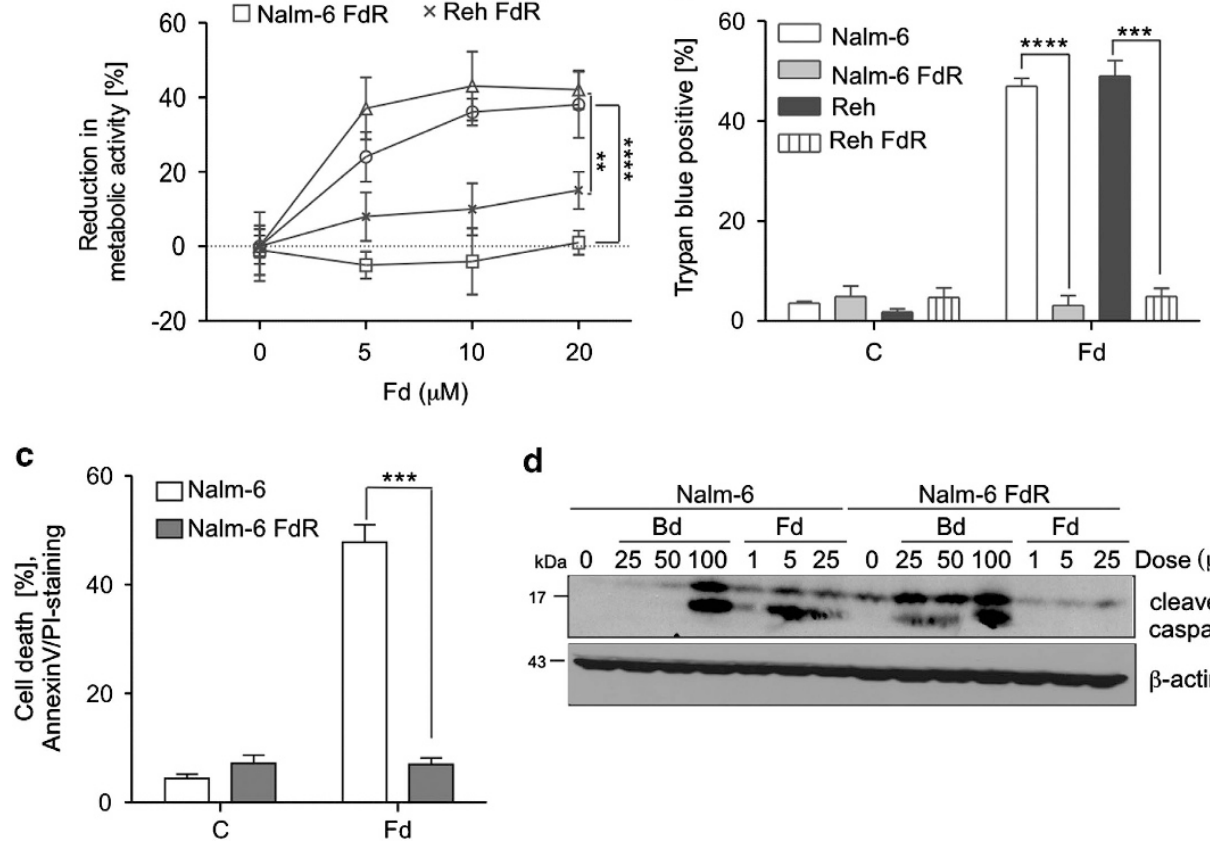

d

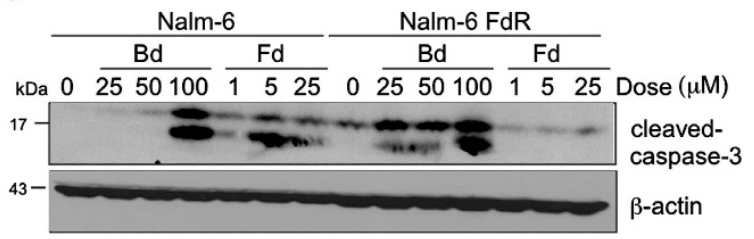

Figure 3 Developing Fd resistance after chronic exposure. (a) Dose reponse for the effect of 24-h-Fd treatment on cell growth in Nalm-6, Nalm-6-FdR, Reh and Reh FdR cells, as determined by the MTS assay. (b) Cell death analysis in the indicated cell lines in response to $24 \mathrm{~h}$ Fd treatment as determined by trypan blue staining. (c) Cell death analysis in Nalm- 6 and Nalm-6 FdR cells in response to $24 \mathrm{~h}$ Fd treatment as determined by AnnexinV/PI staining. All data are expressed as mean \pm S.D. of percentage of cell death from three independent experiments. (d) Western blot analysis for the levels of cleaved caspase-3, as well as $\beta$-actin as a loading control, in Nalm-6 and Nalm-6-FdR cells treated with the indicated concentrations of bendamustine or Fd for $6 \mathrm{~h} ;{ }^{* *} P<0.01,{ }^{* * *} P<0.001,{ }^{* * *} P<0.0001, n=3$

cells, there were LC3 puncta, which colocalized with LAMP2 to a greater extent than in Nalm-6 cells $(P<0.001)$, which were further increased by $C Q(P<0.001)$, suggesting basal autophagy induction and ongoing flux in FdR cells.

Next, we determined the effect of inhibition of autophagy in FdR cells by depleting cells of LAMP2. LAMP2 expression was significantly reduced in shLAMP2- compared with that in shControl-expressing FdR cells (Figure 4d). Moreover, cell death was significantly increased $(P<0.05)$ in shLAMP2expression compared with shControl-expressing FdR cells (Figure 4e). In addition, inhibition of autophagy by shLAMP2 sensitized FdR cells to Fd. Similar findings were obtained upon pharmacological inhibition of autophagy using $C Q$ in FdR cells (Figure 4f). These findings were validated in the CLL-derived cell lines Mec-1 and Mec-2. Consistent with previous studies, ${ }^{26,27}$ both of these cell lines were resistant to $\mathrm{Fd}$, as no significant decrease in MTS reduction was observed even after treatment with up to $150 \mu \mathrm{m}$ Fd for $24 \mathrm{~h}$, with Mec-1 being more resistant (Figure $4 \mathrm{~g}$ and Supplementary Figure $\mathrm{S} 2 \mathrm{~b})$. Importantly, CQ pretreatment sensitized Mec-2 cells to Fd-induced cell death $(P<0.05$; Figure $4 \mathrm{~h})$. CQ treatment alone induced cell death in Mec-2 cells (Figure 4h). These findings further support that these cells are addicted to basal autophagy. Interestingly, Mec-1 were more sensitive to CQ treatment than Mec-2 (Supplementary Figure S2c). This suggests that Mec-1 are more addicted to constitutive autophagy than Mec-2 cells, the latter also being relatively less resistant to $\mathrm{Fd}$. Thus, FdR cells become addicted to higher constitutive autophagy and inhibition of autophagy in FdR cells sensitizes them to Fd.
Sustained MCL-1 expression inhibits BECN1-dependent autophagic cell death in FdR cells. To distinguish between the opposing roles of autophagy in regulating cell death, that is, being prodeath in Fd-sensitive and prosurvival in FdR cells, we next investigated the relationship between autophagy and apoptosis at the molecular level. One of the best studied complexes where components of both autophagy and apoptosis have been known to converge is the multimeric class III PI3K, BECN1/Vps34/Vps15-complex. ${ }^{13,16,17}$ We first examined the levels of various BCL-2 family proteins and BECN1 in Fd-treated Nalm-6 and Reh cells and their resistant derivatives. In both Nalm- 6 and Reh cells we observed downregulation of MCL- 1 within $4 \mathrm{~h}$ of $\mathrm{Fd}$ treatment. In contrast, MCL-1 levels were maintained in the resistant derivatives (Figures $5 \mathrm{a}$ and $\mathrm{b}$ ). Interestingly, BIM levels were greatly reduced and BCL-XL levels were increased in FdR compared with Fd-sensitive cells. Expression of the other BCL-2 family proteins examined was largely unaffected.

Next, we immunoprecipitated MCL- 1 from both Nalm- 6 and FdR cells following Fd treatment and examined the levels of associated BECN1 and BIM. In untreated Nalm- 6 cells, both BECN1 and BIM were bound to MCL-1 (Figure $5 \mathrm{C}$ ). There was no BECN1/MCL-1-complex in Nalm-6 cells following Fd treatment (Figure $5 \mathrm{c}$ ), as expected due to degradation of MCL-1. Thus, BECN1 dissociated and led to the induction of autophagy. Interestingly, there was much more MCL-1 bound to BECN1 in FdR as compared with Nalm-6 cells (Figure $5 \mathrm{c}$ ) suggesting an inhibition of BECN1-mediated autophagy in FdR cells. In addition, BIM was released from MCL-1 following 

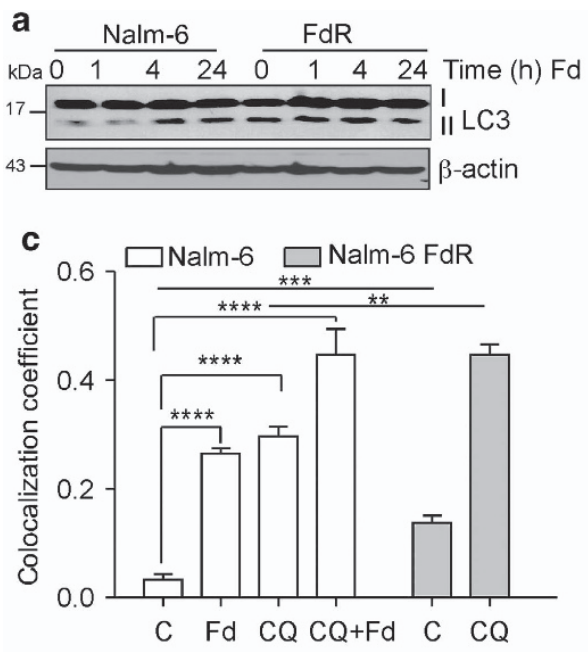

d
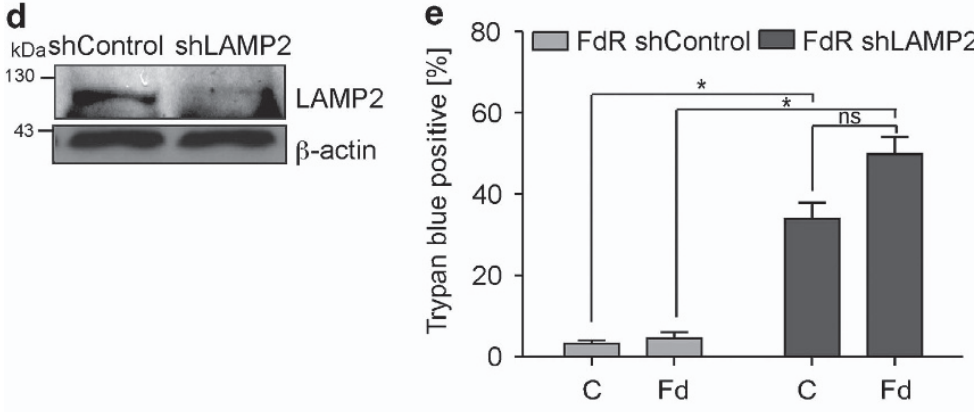

b

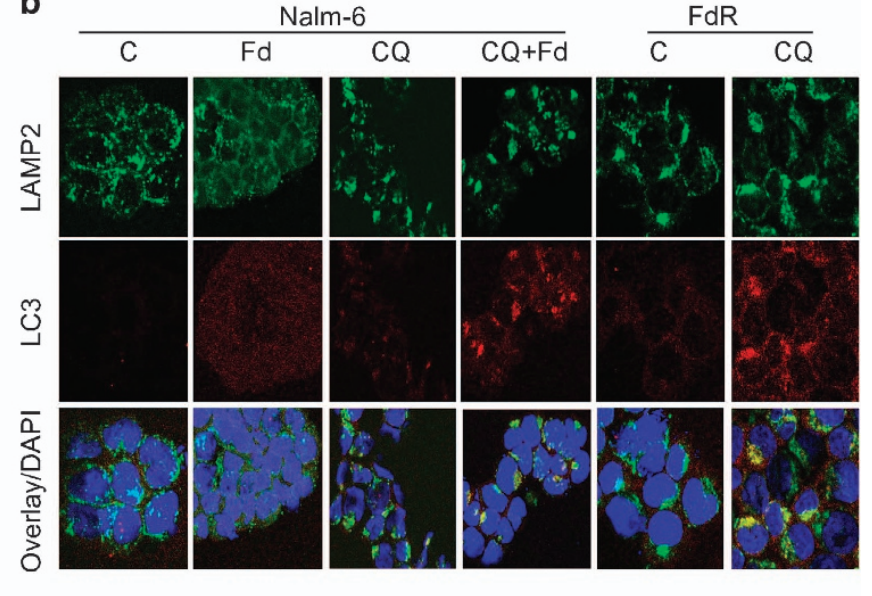

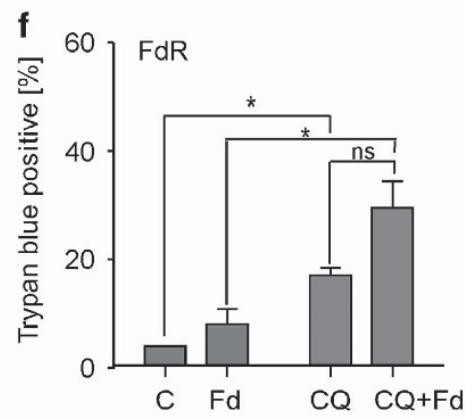

g

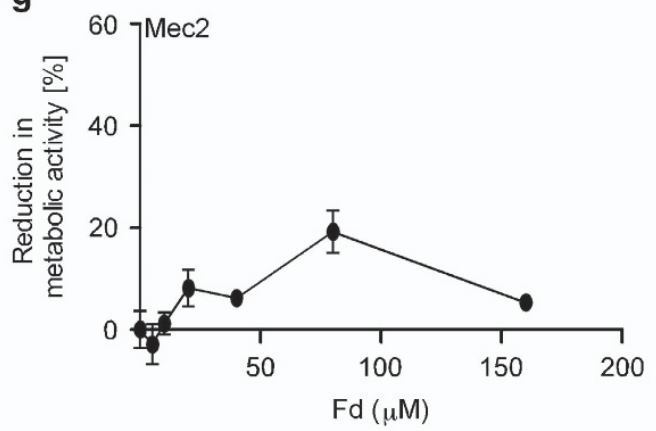

h

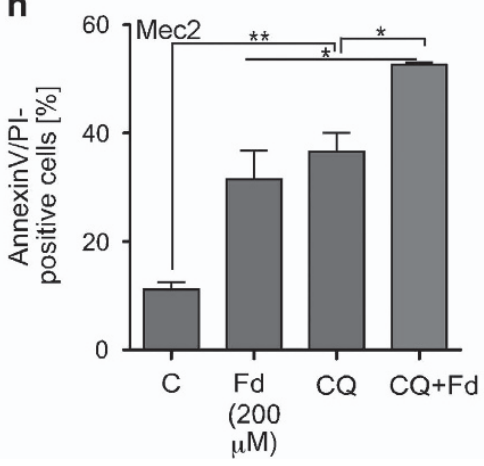

Figure 4 FdR cells are addicted to basal autophagy. (a) Western blot analysis for the levels of LC3 I/II and $\beta$-actin used as a loading control, in Nalm-6 and their derivative FdR cells in response to fludarabine (Fd)-treatment for the indicated time. (b) Nalm-6 and Nalm-6 FdR cells were pretreated with $C Q$ for $1 \mathrm{~h} \pm \mathrm{Fd}(4 \mathrm{~h})$. Cells were then fixed, stained with an antibody against LC3 and LAMP2 along with nuclear staining by DAPI and analyzed by immunofluorescence. (c) Correlation index for LC3 and LAMP2 in the indicated samples. (d) Cell death analysis in ShControl and shLAMP2-expressing Nalm-6 FdR cells in response to $24 \mathrm{~h} F d$ treatment, as determined by trypan blue staining. (e) Western blot analysis for the levels of LAMP2, as well as $\beta$-actin in Nalm-6 FdR cells expressing shControl or shLAMP2. (f) Nalm-6 FdR cells were pretreated with CQ for $1 \mathrm{~h}$ followed by $24 \mathrm{~h} \mathrm{Fd}$ treatment and cell death was determined by AnnexinV/PI staining. (g) Dose reponse for the effect of $24 \mathrm{~h}$ Fd treatment on cell growth in Mec-2 cells, as determined by the MTS assay. (h) Mec-2 cells were pretreated with $C Q$ for $1 \mathrm{~h}$ followed by $24 \mathrm{~h}$ Fd treatment and cell death was determined by AnnexinV/PI staining. All data are expressed as mean \pm S.D. of percentage of cell death from three independent experiments; ${ }^{\star} P<0.05,{ }^{* \star} P<0.01,{ }^{\star \star *} P<0.001,{ }^{* \star *} P<0.0001, n=3$

Fd treatment in Nalm-6 cells (Figure 5c), therefore, was capable of initiating apoptosis. Consistent with the very low levels of BIM in FdR cells (Figure 5b), we did not find any BIM in complex with MCL-1 (Figure 5c). Moreover, Fd-induced cell death was significantly reduced in siBIM- versus siControlexpressing Nalm-6 $(P<0.05)$ cells (Figures $5 f$ and $g$ ). Additionally, we examined BECN1 and BIM interactions with BCL-2 and BCL-XL in Nalm- 6 and FdR cells treated with Fd (Figures $5 d$ and e). In Nalm-6 cells, none of these interactions changed following $\mathrm{Fd}$ treatment. In FdR cells, there was enhanced interaction of BECN1 with both BCL-2 and BCL-XL compared with that in Nalm- 6 cells. These results suggest that BCL-2 family proteins promote cell survival by inhibition of both apoptosis and BECN1-mediated autophagy in FdR cells.

Treatment with obatoclax, a small-molecule antagonist of the $\mathrm{BH} 3$-binding domain of $\mathrm{BCL}-2$ proteins, caused release of both BIM and BECN1 in Fd-sensitive cells and BECN1 in FdR cells from the MCL-1-complex (Figure 5h). Interestingly, 
a

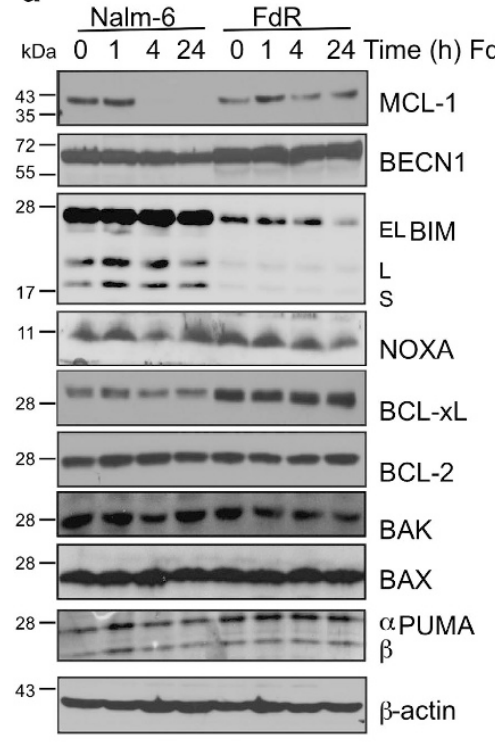

e

Nalm-6 FdR Nalm-6 FdR $\mathrm{kDa} C \mathrm{Fd} C \mathrm{CF} C \mathrm{C} \mathrm{Fd} \mathrm{C} \mathrm{Fd}$

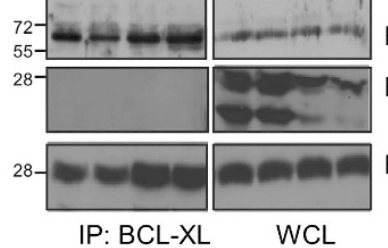

IP: BCL-XL WCL b

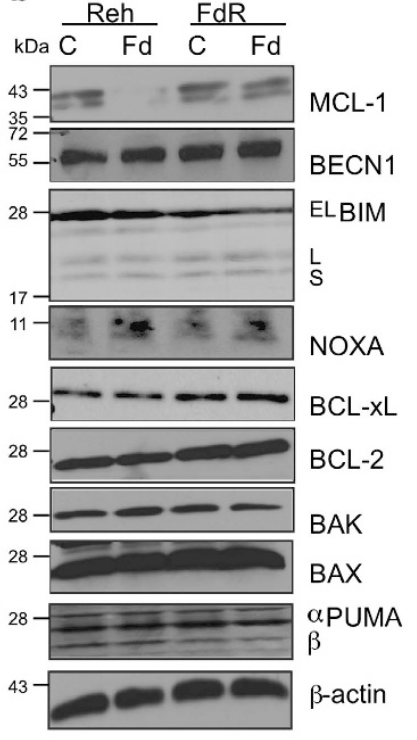

f

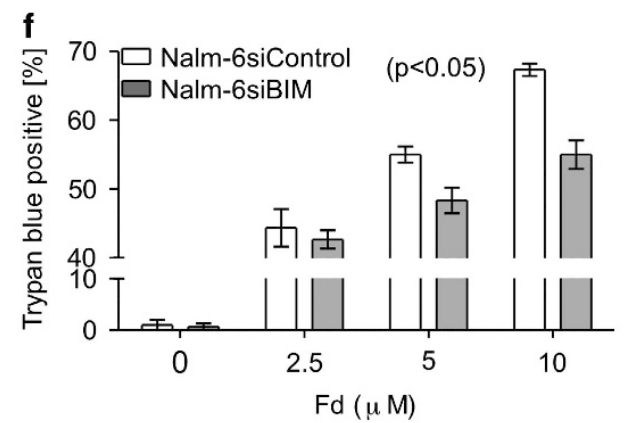

C Nalm-6 FdR Nalm-6 FdR kDa C Fd C FdlgG C Fd C Fd

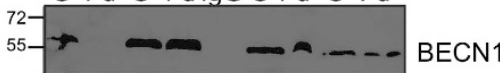

$28-\square$
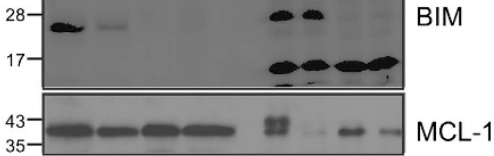

IP: MCL-1 WCL

d

Nalm-6 FdR Nalm-6 FdR $\overline{C F d} \overline{C F d} \overline{C F d} \overline{C ~ F d}$

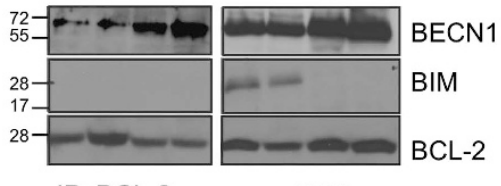

IP: BCL-2 WCL h

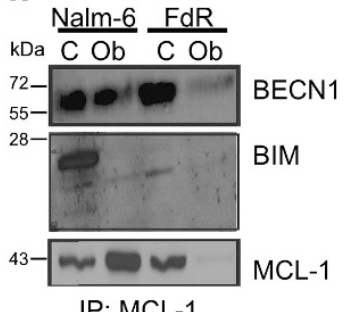

i

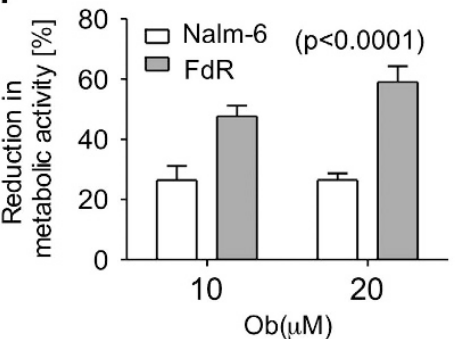

g

Nalm-6

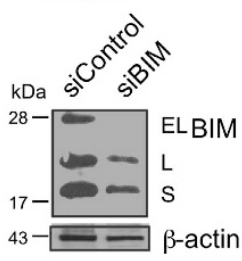

j

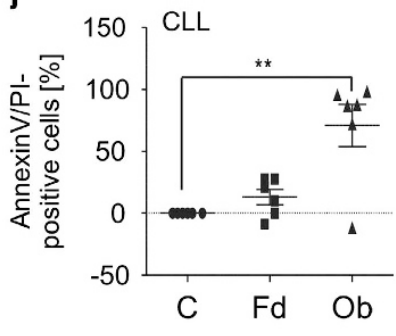

Figure 5 Sustained MCL-1 expression inhibits BECN1-dependent autophagic cell death in FdR cells. (a) Nalm-6 and Nalm-6 FdR, as well as (b) Reh and Reh FdR cells were treated with $\mathrm{Fd}$ for the indicated time and $4 \mathrm{~h}$, respectively. Cell lysates were analyzed by western blotting for the indicated proteins. $\beta$-actin was used as a loading control. Nalm-6 and Nalm-6 FdR cells were treated with Fd for $24 \mathrm{~h}$, and (c) MCL-1, (d) BCL-2 and (e) BCL-XL immunoprecipitates and whole cell lysates (WCL) were analyzed for the presence of proteins with the corresponding primary antibodies. (f) Cell death analysis in siControl and siBIM-expressing Nalm- 6 cells in response to $24 \mathrm{~h}$ Fd treatment as determined by trypan blue staining. (g) Immunoblot analysis of BIM and $\beta$-actin as a loading control in Nalm-6 cells expressing siControl or siBIM. (h) Nalm-6 and Nalm-6 FdR cells were treated with obatoclax (ob) for $24 \mathrm{~h}$, and MCL-1 immunoprecipitates were analyzed for the presence of the indicated proteins. (i) Nalm- 6 and Nalm-6 FdR cells were treated with increasing concentrations of ob for $24 \mathrm{~h}$ and the effect on cell viability was assessed by the MTS assay. (j) CLL cells were treated with Fd \pm ob ( $5 \mu \mathrm{M}$ ) for $48 \mathrm{~h}$ and cell death was determined by AnnexinV/PI staining; ${ }^{* *} P<0.01, n=6$

while in the Fd-sensitive cells obatoclax did not cause a decrease in MCL-1 levels, in FdR cells the release of BECN1 from the MCL-1-complex was at least, in part, due to degradation of MCL-1 (data not shown). Importantly, Nalm6- and Nalm-6-FdR cells were both sensitive to obatoclax, validating the importance of functional $B C L-2$ family proteins in FdR cells (Figure 5i). Moreover, in primary CLL cells 24-hobatoclax treatment significantly increased $(P<0.01)$ the
AnnexinV/PI-positive cell population (Figure 5j). Thus, degradation of MCL-1 in Fd-sensitive cells released BECN1 and BIM from the MCL-1-complex and, therefore, autophagy and apoptosis occurred simultaneously. In contrast, in FdR cells low-BIM levels prevented apoptosis. In addition, BCL-2, BCL-XL and stabilized MCL-1 kept BECN1 sequestered and prevented activation of autophagy-associated with cell death. Importantly, inhibition of BCL-2 family by obatoclax could 
target both Fd-sensitive cells by induction of BIM-dependent apoptosis and BECN1-dependent autophagy and FdR cells by induction of BECN1-dependent autophagy.

\section{Basal autophagy in FdR cells is dependent on AMPK but} not BECN1. To reconcile our findings that cell deathassociated but not basal autophagy was inhibited in FdR cells, with reports of BECN1-independent autophagy, ${ }^{28}$ we next examined whether basal autophagy in FdR cells was independent of BECN1. Immunoblot analysis indicated a significant reduction in BECN1 levels in siBECN1- compared with siControl-expressing Nalm-6 and FdR cells (Figure 6a). In siBECN1-expressing Nalm-6, Fd-induced LC3 processing was decreased and the levels of p62/SQSTM1 were higher in both untreated and Fd-treated cells compared with siControlexpressing cells (Figure 6a), suggesting that both basal and Fd-induced autophagy is BECN1-dependent. However, there was no change in LC3 processing or p62/SQSTM1 levels in FdR cells depleted of BECN1 (Figure 6a), further supporting BECN1-independent basal autophagy in FdR cells. Moreover, Fd-induced cell death was significantly reduced in
siBECN1- $(P<0.001)$ compared with siControl-expressing Nalm-6 cells (Figure $6 \mathrm{~b}$ ). This was consistent with what we have shown earlier (Figures 2 and 5) that is, Fd induces BECN1-dependent autophagy-associated cell death in Nalm6 cells. Interestingly, the presence or absence of BECN1 made no difference to cell survival in $\mathrm{Fd}$ treated or untreated FdR cells (Figure 6b). These findings further substantiate that basal autophagy in FdR cells is BECN1-independent, such that inhibition of autophagy by LAMP2, but not BECN1 knockdown causes cell death (Figure 4e). Importantly, obatoclax-induced autophagy was reduced in siBECN1FdR compared with that in siControl-FdR cells, as determined by p62/SQSTM1 levels, supporting the observation that obatoclax relieves $\mathrm{MCL}-1$-mediated inhibition on BECN1 in FdR cells, thus leading to BECN1-dependent autophagy (Figure 6c).

Under energy stress conditions, the AMP-activated protein kinase (AMPK) activates autophagy by direct phosphorylation of Unc51-like kinase (ULK-1), which forms a multi-molecularcomplex associated with initiation of autophagy. ${ }^{29}$ Interestingly, FdR cells showed increased sensitivity to serum
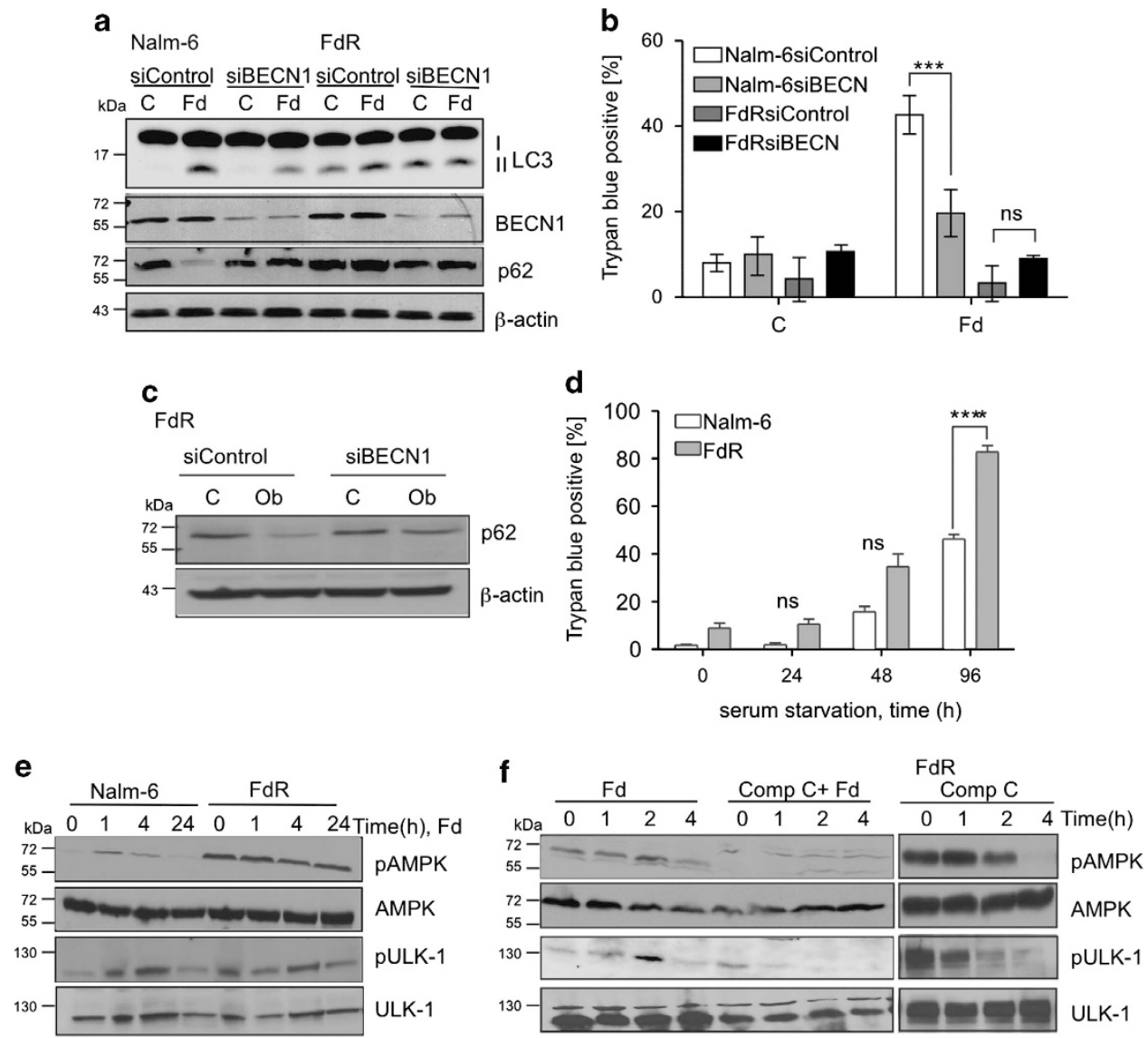

Figure 6 Basal autophagy in FdR cells is dependent on AMPK but not BECN1. (a) Immunoblot analysis of LC3, BECN1, p62 and $\beta$-actin as a loading control in Nalm-6 and Nalm-6 FdR cells expressing siControl or siBECN1 treated with Fd for $4 \mathrm{~h}$. (b) Cell death analysis in siControl and siBECN1-expressing cells in response to $24 \mathrm{~h}$ Fd treatment as determined by trypan blue staining ${ }^{\star} P<0.01, n=3$. (c) Immunoblot analysis of $p 62$ and $\beta$-actin as a loading control in Nalm-6 FdR cells expressing siControl or siBECN1 treated with obatoclax for $24 \mathrm{~h}$. (d) Cell death analysis in Nalm 6 and FdR Cells in response to serum starvation for the indicated time, as determined by trypan blue staining, ${ }^{* * * *} P<0.0001, n=3$. (e) Nalm- 6 and Nalm 6 FdR cells were treated with Fd for the indicated time. Cell lysates were analyzed by western blotting for pAMPK-Thr172, AMPK, pULK-1-Ser467 and ULK-1. (f) Nalm-6 and Nalm-6 FdR cells were treated with Comp C \pm Fd and Comp C, respectively, for the indicated time and analyzed by western blotting for the levels of pAMPK-Thr172, AMPK, pULK-1-Ser467 and ULK-1 


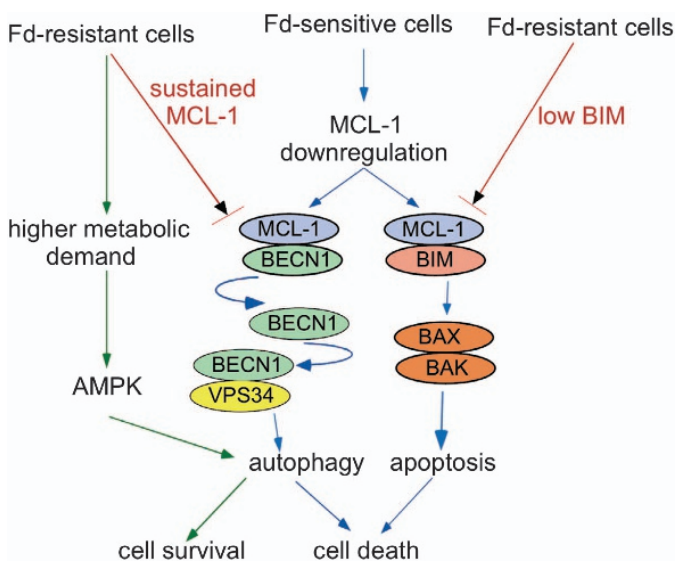

Figure 7 Model for acquired Fd resistance mechanism. MCL-1 degradation following Fd treatment released BIM and BECN1, leading to apoptosis and cell death-associated autophagy in $\mathrm{Fd}$-sensitive cells. In FdR cells there was low-BIM expression and BECN1 was inhibited by high MCL-1/BECN1 interaction. FdR cells have a higher metabolic demand, which activates AMPK-dependent autophagy and promotes survival in these cells

starvation (Figure 6d) and higher basal AMPK and ULK-1 activation compared with Nalm-6 cells (Figure 6e), suggesting higher metabolic stress in FdR cells. In addition, Fd caused AMPK and ULK-1 activation in Nalm-6 and Reh cells (Figure 6e; Supplementary Figure S3), suggesting that Fdinduced autophagy is mediated by AMPK. Importantly, inhibition of AMPK in Nalm- 6 and FdR cells by compound $C$ (Comp C) inhibited autophagy, as determined by pULK-1 levels (Figure 6f). Thus, Fd-induced autophagy was AMPKand BECN1-dependent in Fd-sensitive cells. However, basal autophagy in FdR cells, to which they were addicted as a result of increased metabolic demand, was dependent on AMPK, but not BECN1. Taken together, these data establish that autophagy is critical for regulating cell death and resistance to $\mathrm{Fd}$. Inhibition of BECN1-dependent autophagy, reduced levels of BIM, and AMPK-dependent basal autophagy are key determinants of acquired resistance to $\mathrm{Fd}$ (Figure 7).

\section{Discussion}

Here we show that autophagy is a critical regulator of cellular response to $\mathrm{Fd}$ in leukemic B cells. Autophagy represents an important regulatory mechanism of cellular response to adverse stimuli. Although the protective function of autophagy in response to cellular stress is well accepted, the role of autophagy as a pro-death mechanism is controversial. ${ }^{30,31}$ Our data suggest that autophagy differentially regulates Fdinduced cell death in sensitive cells and those with acquired Fd resistance. Thus, Fd induces autophagy and enhances autophagic flux in Fd-sensitive cells, as previously reported in CLL cells. ${ }^{32}$ Additionally, we reveal for the first time that Fd-induced autophagy is BECN1-dependent and is associated with cell death in Fd-sensitive cells. Inhibition of autophagy using pharmacological (3-MA) and genetic (siBECN1, shATG7 or shLAMP2) means prevented Fdmediated cell death in sensitive cells.
On the basis of our data on the prodeath function of autophagy in Fd-sensitive cells, we expected a complete inhibition of autophagy in FdR cells. Surprisingly, FdR cells showed markers of increased constitutive autophagy: higher LC3-II expression, LC3 puncta formation and fusion between autophagosomes and lysosomes. Additionally, we found that the higher basal autophagy was essential for the survival of FdR cells, including the CLL cell lines Mec-1 and Mec-2, as cell death increased upon autophagy inhibition. These findings indicate that other adaptive changes might have happened during the chronic exposure to Fd during selection for Fd resistance, which contributed to overcome cell death despite increased autophagy.

Importantly, in Fd-sensitive cells $\mathrm{MCL}-1$ degradation following $\mathrm{Fd}$ treatment released BECN1 from the MCL1-complex to cause autophagy-associated cell death. In contrast, in FdR cells MCL- 1 levels were sustained, leading to sequestration of BECN1 and inhibition of BECN1-mediated autophagy. Although the BCL-2 family has been implicated in the regulation of apoptosis and $\mathrm{Fd}$ resistance in CLL, and of autophagy in other systems, ${ }^{16,33,34}$ to our knowledge this is the first report that demonstrates the role of BCL-2 family in regulation of autophagy, in addition to apoptosis, in response to $\mathrm{Fd}$.

BCL-2 family members, especially MCL-1, have an important role in regulating in vitro drug resistance in CLL. ${ }^{6,35}$ Importantly, the BIM-MCL-1-complex is known to be critical for apoptosis modulation in CLL. ${ }^{36}$ We show that endogenous MCL-1 sequestered BIM in untreated Fdsensitive cells to inhibit apoptosis. Fd treatment reduced MCL-1 levels and released BIM to initiate apoptosis. Interestingly, FdR cells had remarkably low-BIM levels, which at least, in part, were regulated transcriptionally (data not shown). Thus, we show for the first time that levels of BIM and its interaction with $\mathrm{MCL}-1$ are important determinants of $\mathrm{Fd}$ mediated apoptosis.

Induction of cell death by the pan-Bcl-2 inhibitor obatoclax in both $\mathrm{Fd}$-sensitive and $\mathrm{FdR}$ cells underscores the regulation of multiple cell death pathways by BCL-2 family proteins. Clearly, obatoclax induced BIM and BECN1 release from the MCL-1-complexes in Fd-sensitive cells. However, in FdR cells, which have no BIM (apoptotic factor), obatoclax induced BECN1 release from MCL-1 and other BCL-2 family proteins. Free BECN1 is then able to induce autophagyassociated cell death.

We found that FdR cells exhibit increased sensitivity to serum starvation compared with Fd-sensitive cells. The increase in metabolic demand may, in part, explain the increased dependence of FdR cells to basal autophagy. Our data suggest that basal autophagy in $\mathrm{FdR}$, but not in $\mathrm{Fd}$ sensitive cells, was independent of BECN1. siBECN1expressing Fd-sensitive cells showed reduced autophagy and cell death in the presence of $\mathrm{Fd}$, whereas siBECN1expression in FdR cells had no effect on basal or Fd-induced autophagy or cell survival.

Previous studies have suggested that AMPK activation, a sensor of the cellular energy status, stimulates autophagy via AMPK-mediated phosphorylation of ULK-1. ${ }^{29}$ Consistently, we found AMPK activation in response to Fd in sensitive cells and that AMPK activation was remarkably higher in FdR 
compared with Fd-sensitive cells. In addition, activation of AMPK corresponded to ULK-1 phosphorylation at Ser467, one of the AMPK target sites on ULK-1. ${ }^{29}$ Moreover, inhibition of AMPK prevented ULK-1 activation. These results suggest that AMPK has a critical role in Fd-mediated autophagy.

Fd resistance has been often associated with loss of p53. ${ }^{37}$ We found that both $\mathrm{Fd}$ and ionizing radiation (IR), a prototypical DNA damaging reagent, ${ }^{38}$ induced p53 in Fd-sensitive, but not resistant cells (Supplementary Figures $4 a$ and b). In addition, p21, a downstream target of p53, was robustly induced by both $\mathrm{Fd}$ and IR in sensitive cells, whereas FdR cells showed a delayed induction in response to IR (Supplementary Figure 4c), suggesting an attenuated p53 function in FdR cells. Importantly, any perturbation of p53 pathway - either activation or inhibition - has been reported to induce autophagy. p53 is known to activate BECN1-dependent autophagy through JNK-mediated phosphorylation of BCL-2. ${ }^{39}$ Besides, cytoplasmic p53 is well established to inhibit autophagy..$^{40}$ In our preliminary experiments, pifithrin- $\alpha$, a transcriptional inhibitor of $\mathrm{p} 53^{41}$ did not affect Fd-mediated autophagy in Nalm- 6 cells although p21 upregulation was inhibited (Supplementary Figure 4d). However, regulation of autophagy by $\mathrm{p} 53$ would require more detailed investigations that are beyond the scope of this study. Nevertheless, from our data it is conceivable that in FdR cells p53 attenuation may be associated with both inhibition of cytotoxic (BECN1dependent) and activation of cytoprotective autophagy (AMPK/ULK-1-dependent). Therefore, inhibition of autophagy may possibly be a treatment strategy for CLL patients with defective p53.

In conclusion, our data establish that autophagy can have a differential outcome on cell death or cell survival. In Fdsensitive cells, a BECN1-dependent signaling mechanism mediates autophagic cell death in response to $\mathrm{Fd}$, whereas FdR cells become addicted to basal autophagy that is required for their cell survival. This transition is achieved by simultaneous increase in the MCL-1/BECN1 interaction to inhibit cell death-associated BECN1-dependent autophagy, as well as activation of BECN1-independent and AMPKdependent autophagy. In addition to autophagy, BIM-dependent apoptosis is critical for cell death due to Fd and FdR cells escape this cell death pathway by regulating BIM levels. These data suggest that elevated basal autophagy or low-BIM levels, such as that seen in FdR cells may be useful to predict patient response to chemotherapy and/or autophagy inhibition. These findings have important implications in designing novel therapeutic regimens for CLL patients. Although induction of autophagy may be a useful strategy to induce cell death in Fd-sensitive patients, these findings suggest that paradoxically, inhibition of autophagy may be a viable treatment strategy for CLL patients that have demonstrated Fd resistance.

\section{Materials and Methods}

Reagents and plasmids. Fd (9- $\beta$-D-arabinofuranosyl-2-fluoroadenine 5 '-phosphate), CQ, 3-methyladenine and Comp $C$ were purchased from Sigma Aldrich (St. Louis, MO, USA). pLKO.1-puro control vector, shATG7 (cat no. SHCLNG-NM_006395) and shLAMP2 (cat no. SHCLNG-NM_002294) Mission shRNAs from Sigma Aldrich and packaging plasmids pVSVG and dr 8.7 from Invitrogen (Carlsbad, CA, USA). Lentiviral infections were performed using polybrene $(10 \mu \mathrm{g} / \mathrm{ml})$ from Sigma Aldrich, according to manufacturer's instructions. Cells were treated with $10 \mu \mathrm{m} \mathrm{Fd}, 25 \mu \mathrm{m} \mathrm{CQ}, 10 \mathrm{~mm}$ 3-MA, $10 \mu \mathrm{m}$ obatoclax (Selleck, Houston, TX, USA) and $1 \mu \mathrm{m}$ Comp C, $10 \mu \mathrm{m}$ Cyclic Pifithrin- $\alpha$ hydrobromide, unless otherwise stated.

Cell lines and patient samples. Human preB acute lymphocytic leukemic cell lines, Nalm-6 and Reh cells were obtained from ATCC (Manassas, VA, USA). $\mathrm{FdR}$ cells were generated by initially culturing cells with lower concentration of $\mathrm{Fd}$ for short periods of time followed by $48 \mathrm{~h}$ of recovery time. The concentration of the drug was increased gradually until the desired resistance, twice the $\mathrm{IC}_{50}$ value, was achieved. The resistant cells were intermittently treated with verapamil (Sigma Aldrich) to eliminate the chance of acquired resistance due to increased expression of drug efflux pumps. In addition to these derivatized FdR cells, we also used the Mec-1 and Mec-2 (a gift from Dr. Y. Saunthararajah (Cleveland Clinic)), CLL-derived cell lines, known to be inherently resistant to $\mathrm{Fd}^{26,27}$ Cells were maintained in RPMI-1640 medium supplemented with $10 \%$ fetal bovine serum (Atlanta Biologicals, Lawrenceville, GA, USA), L-glutamine (Gibco BRL, Gaithersburg, MD, USA) and Antibiotic-antimycotic (Invitrogen).

Peripheral blood samples were obtained from CLL patients after informed consent according to protocols approved by the institutional review board (IRB) according to the Declaration of Helsinki. Briefly, lymphocytes from blood samples were purified by Ficoll-Paque PLUS (Amersham Biosciences, Piscataway, NJ, USA) gradient centrifugation. Primary cells were cultured and cell death was assayed as previously described. ${ }^{4,42}$

Radiation treatment. IR was delivered, as described, ${ }^{8,9}$ using a conventional cesium-137 $\gamma$-irradiator (JL Shepherd Associates, San Fernando, CA, USA), at a dose rate of $149 \mathrm{cGy} / \mathrm{min}$.

Immunoblotting and immunoprecipitation. Cell lysates for immunoblotting and immunoprecipitation were prepared, as described previously. ${ }^{5}$ The primary antibodies were used against: LC3, pAMPK (Thr172), AMPK, pULK-1 (Ser467), ULK-1, cleaved caspase-3, ATG7 (Cell Signaling Technologies, Danvers, MA, USA); MCL-1, BIM (BD Biosciences, San Jose, CA, USA); NOXA (Enzo Life Sciences, Farmingdale, NY, USA); p62 (Fitzgerald Industries international, Acton, MA, USA); LAMP2, BCL-2, BCL-XL, BECN1, BAX, p21, p53 (Santa Cruz Biotechnologies, Santa Cruz, CA, USA); BAK (Calbiochem, Billerica, MA, USA); $\beta$-actin (Sigma Aldrich); PUMA (Prosci Incorporated, Poway, CA, USA); secondary anti-mouse HRP (Millipore, Danvers, MA, USA); and secondary anti-rabbit HRP (Fisher Scientific, Pittsburg, PA, USA).

Confocal immunostaining. Treated cells were washed with PBS and cytoslides were prepared. The slides were then fixed with 2.0\% paraformaldehyde/ PBS for 15 min at room temperature, permeabilized with $0.1 \%$ Triton X-100 in PBS for $5 \mathrm{~min}$, blocked in $10 \%$ fetal bovine serum and $0.1 \%$ Triton X-100 in PBS for $1 \mathrm{~h}$ and incubated with the antibodies of interest diluted in blocking buffer for $1 \mathrm{~h}$, followed by $1 \mathrm{~h}$ incubation with fluorescently conjugated secondary antibody, and finally with $4^{\prime}, 6$-diamidino-2-phenylindole to stain nuclei. They were then mounted in Vectashield (Vector Laboratories, Burlingame, CA, USA). Images were collected using an HCX Plan Apo 63X/1.4N.A. oil immersion objective lens on a Leica TCSSP2 confocal microscope (Leica Microsystems AG, Buffalo Grove, IL, USA). The average LC3 puncta per cell was determined using Image $\mathrm{J}$ software $(\mathrm{NIH}$, Bethesda, MD, USA). For LAMP2/LC3 colocalization, Pearson correlation coefficient was calculated using Image-Pro Plus software $(\mathrm{NIH})$. For all image quantifications, data were collected from at least 30 cells.

Cell viability and apoptosis assays. 3-(4,5-dimethylthiazol-2-yl)-5-(3carboxymethoxyphenyl)-2-(4-sulfophenyl)-2H tetrazolium inner salt (MTS) assay (Promega, Madison, WI, USA) was used to assess cell proliferation. Data are expressed as \% reduction in metabolic activity that is, $100-((0 . D .490 \mathrm{~nm}$ Untreated) $-(0 . D .490 \mathrm{~nm}$ Treated) $) /\left(0 . D .490 \mathrm{~nm}\right.$ Untreated) $\left.{ }^{*} 100\right)$ versus the indicated concentrations of the drug. Cell death was measured by trypan blue staining. Apoptosis was measured using AnnexinV-fluorescein isothiocyanate and propidium iodide staining (BD Biosciences), as described previously. ${ }^{38}$ Cell death data were acquired on a BD FACS Calibur flow cytometer (BD Biosciences) and analyzed by CellQuest software (BD Biosciences, Franklin Lakes, NJ, USA).

Statistical analysis. Statistical comparisons between two groups were conducted by using the Student's $t$-test and between multiple groups using 
two-way ANOVA using the (Prism software GraphPad Software, Inc., La Jolla, CA USA). Error bars indicate S.D., which was calculated from three independent experiments performed in triplicates.

\section{Conflict of Interest}

The authors declare no conflict of interest.

Acknowledgements. This work was supported by a research grant from National Institutes of Health (CA127264; awarded to AA), a US Department of Defense Post-doctoral Training Award (PC094405; awarded to KS) and a fellowship from Cleveland State University (Molecular Medicine Program to AS).

1. Van den Neste E, Cardoen S, Offner F, Bontemps F. Old and new insights into the mechanisms of action of two nucleoside analogs active in lymphoid malignancies: fludarabine and cladribine. Int J Oncol 2005; 27: 1113-1124.

2. Gribben JG, O'Brien S. Update on therapy of chronic lymphocytic leukemia. J Clin Oncol 2011; 29: 544-550.

3. Kelly PN, Strasser A. The role of Bcl-2 and its pro-survival relatives in tumourigenesis and cancer therapy. Cell Death Differ 2011; 18: 1414-1424.

4. Al-Harbi S, Hill BT, Mazumder S, Singh K, Devecchio J, Choudhary G et al. An antiapoptotic BCL-2 family expression index predicts the response of chronic lymphocytic leukemia to ABT-737. Blood 2011; 118: 3579-3590.

5. Mazumder S, Choudhary GS, Al-Harbi S, Almasan A. Mcl-1 Phosphorylation defines ABT737 resistance that can be overcome by increased NOXA expression in leukemic $B$ cells. Cancer Res 2012; 72: 3069-3079.

6. Pepper C, Lin TT, Pratt G, Hewamana S, Brennan P, Hiller L et al. Mcl-1 expression has in vitro and in vivo significance in chronic lymphocytic leukemia and is associated with other poor prognostic markers. Blood 2008; 112: 3807-3817.

7. Garcia-Saez AJ. The secrets of the Bcl-2 family. Cell Death Differ 2012; 19: 1733-1740.

8. Chen $Q$, Gong B, Almasan A. Distinct stages of cytochrome $c$ release from mitochondria: evidence for a feedback amplification loop linking caspase activation to mitochondrial dysfunction in genotoxic stress induced apoptosis. Cell Death Differ 2000; 7: 227-233.

9. Salvesen GS, Ashkenazi A. Snapshot: caspases. Cell 2011; 147: 476-476 e471.

10. Youle RJ, Strasser A. The BCL-2 protein family: opposing activities that mediate cell death. Nat Rev Mol Cell Biol 2008; 9: 47-59.

11. Hardwick JM, Chen YB, Jonas EA. Multipolar functions of BCL-2 proteins link energetics to apoptosis. Trends Cell Biol 2012; 22: 318-328.

12. Kang R, Zeh HJ, Lotze MT, Tang D. The Beclin 1 network regulates autophagy and apoptosis. Cell Death Differ 2011; 18: 571-580.

13. Levine B, Sinha S, Kroemer G. Bcl-2 family members: dual regulators of apoptosis and autophagy. Autophagy 2008; 4: 600-606.

14. Klionsky DJ, Abdalla FC, Abeliovich H, Abraham RT, Acevedo-Arozena A, Adeli K et al. Guidelines for the use and interpretation of assays for monitoring autophagy. Autophagy 2012; 8: 445-544.

15. Yorimitsu T, Klionsky DJ. Autophagy: molecular machinery for self-eating. Cell Death Differ 2005; 12(Suppl 2): 1542-1552.

16. Bonapace L, Bornhauser BC, Schmitz M, Cario G, Ziegler U, Niggli FK et al. Induction of autophagy-dependent necroptosis is required for childhood acute lymphoblastic leukemia cells to overcome glucocorticoid resistance. J Clinical Invest 2010; 120: 1310-1323.

17. Marquez RT, Xu L. Bcl-2:Beclin 1 complex: multiple, mechanisms regulating autophagy/ apoptosis toggle switch. Am J Cancer Res 2012; 2: 214-221.

18. Chaachouay H, Ohneseit P, Toulany M, Kehlbach R, Multhoff G, Rodemann HP. Autophagy contributes to resistance of tumor cells to ionizing radiation. Radiother Oncol 2011; 99: 287-292

19. Li H, Wang P, Sun Q, Ding WX, Yin XM, Sobol RW et al. Following cytochrome c release, autophagy is inhibited during chemotherapy-induced apoptosis by caspase 8-mediated cleavage of Beclin 1. Cancer Res 2011; 71: 3625-3634.

20. Gao W, Shen Z, Shang L, Wang X. Upregulation of human autophagy-initiation kinase ULK1 by tumor suppressor p53 contributes to DNA-damage-induced cell death. Cell Death Differ 2011; 18: 1598-1607.

21. Laane E, Tamm KP, Buentke E, Ito K, Kharaziha P, Oscarsson J et al. Cell death induced by dexamethasone in lymphoid leukemia is mediated through initiation of autophagy. Cell Death Differ 2009; 16: 1018-1029.
22. Kabeya Y, Mizushima N, Uero T, Yamamoto A, Kirisako T, Noda T et al. LC3, a mammalian homologue of yeast Apg8p, is localized in autophagosome membranes after processing EMBO J 2000; 19: 5720-5728.

23. Bjorkoy G, Lamark T, Brech A, Outzen H, Perander M, Overvatn A et al. p62/SQSTM1 forms protein aggregates degraded by autophagy and has a protective effect on huntingtininduced cell death. J Cell Biol 2005; 171: 603-614.

24. Gonzalez-Polo RA, Boya P, Pauleau AL, Jalil A, Larochette N, Souquere $S$ et al. The apoptosis/autophagy paradox: autophagic vacuolization before apoptotic death. J Cell Sci 2005; 118: 3091-3102

25. Maiuri MC, Zalckvar E, Kimchi A, Kroemer G. Self-eating and self-killing: crosstalk between autophagy and apoptosis. Nat Rev Mol Cell Biol 2007; 8: 741-752.

26. Stacchini A, Aragno M, Vallario A, Alfarano A, Circosta P, Gottardi D et al. MEC1 and MEC2: two new cell lines derived from B-chronic lymphocytic leukaemia in prolymphocytoid transformation. Leuk Res 1999; 23: 127-136.

27. Cassano C, Mactier S, Mulligan SP, Belov L, Huang P, Christopherson RI. Cladribine and fludarabine nucleoside change the levels of $C D$ antigens on B-lymphoproliferative disorders. Int J Proteomics 2010; 2010: 964251.

28. Juenemann K, Reits EA. Alternative macroautophagic pathways. Int J Cell Biol 2012; 2012: 189794.

29. Egan DF, Shackelford DB, Mihaylova MM, Gelino S, Kohnz RA, Mair W et al. Phosphorylation of ULK1 (hATG1) by AMP-activated protein kinase connects energy sensing to mitophagy. Science 2011; 331: 456-461.

30. Levine B, Yuan J. Autophagy in cell death: an innocent convict? J Clin Invest 2005; 115 2679-2688.

31. Denton D, Nicolson S, Kumar S. Cell death by autophagy: facts and apparent artefacts. Cell Death Differ 2012; 19: 87-95.

32. Mahoney E, Lucas DM, Gupta SV, Wagner AJ, Herman SE, Smith LL et al. ER stress and autophagy: new discoveries in the mechanism of action and drug resistance of the cyclindependent kinase inhibitor flavopiridol. Blood 2012; 120: 1262-1273.

33. Pattingre S, Tassa A, Qu X, Garuti R, Liang XH, Mizushima N et al. Bcl-2 antiapoptotic proteins inhibit Beclin 1-dependent autophagy. Cell 2005; 122: 927-939.

34. Maiuri MC, Le Toumelin G, Criollo A, Rain JC, Gautier F, Juin P et al. Functional and physical interaction between $\mathrm{Bcl}-\mathrm{X}(\mathrm{L})$ and a BH3-like domain in Beclin-1. EMBO J 2007; 26: 2527-2539

35. Bannerji R, Kitada S, Flinn IW, Pearson M, Young D, Reed JC et al. Apoptotic-regulatory and complement-protecting protein expression in chronic lymphocytic leukemia: relationship to in vivo rituximab resistance. J Clin Oncol 2003; 21: 1466-1471.

36. Paterson A, Mockridge Cl, Adams JE, Krysov S, Potter KN, Duncombe AS et al. Mechanisms and clinical significance of BIM phosphorylation in chronic lymphocytic leukemia. Blood 2012; 119: 1726-1736.

37. Zenz T, Krober A, Scherer K, Habe S, Buhler A, Benner A et al. Monoallelic TP53 inactivation is associated with poor prognosis in chronic lymphocytic leukemia: results from a detailed genetic characterization with long-term follow-up. Blood 2008; 112: 3322-3329.

38. Chen Q, Chai YC, Mazumder S, Jiang C, Macklis RM, Chisolm GM et al. The late increase in intracellular free radical oxygen species during apoptosis is associated with cytochrome c release, caspase activation, and mitochondrial dysfunction. Cell Death Differ 2003; 10: 323-334.

39. Park KJ, Lee SH, Lee CH, Jang JY, Chung J, Kwon MH et al. Upregulation of Beclin-1 expression and phosphorylation of Bcl-2 and p53 are involved in the JNK-mediated autophagic cell death. Biochem Biophys Res Commun 2009; 382: 726-729.

40. Tasdemir E, Maiuri MC, Galluzzi L, Vitale I, Djavaheri-Mergny M, D'Amelio M et al. Regulation of autophagy by cytoplasmic p53. Nat Cell Biol 2008; 10: 676-687.

41. Komarov PG, Komarova EA, Kondratov RV, Christov-Tselkov K, Coon JS, Chernov MV et al. A chemical inhibitor of $\mathrm{p} 53$ that protects mice from the side effects of cancer therapy. Science 1999; 285: 1733-1737.

42. Bucur O, Stancu AL, Khosravi-Far R, Almasan A. Analysis of apoptosis methods recently used in Cancer Research and Cell Death \& Disease publications. Cell Death Dis 2012; 3: e263.

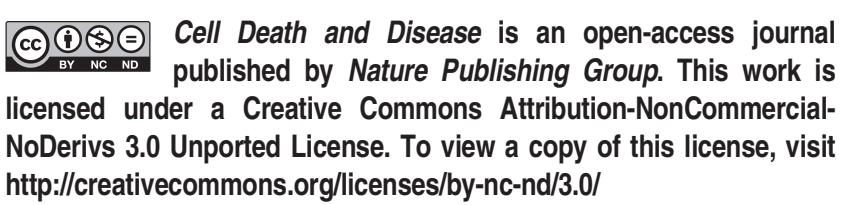

